\title{
EVALUATION OF DIFFERENT APPROACHES FOR THE OPTIMIZATION OF LAYOUT AND CONTROL OF BOOSTER STATIONS
}

\author{
Philipp Pöttgen, and Peter F. Pelz \\ Technische Universität Darmstadt, Chair of Fluid Systems \\ Otto-Berndt-Straße 2, 64283 Darmstadt, Germany \\ \{philipp.poettgen, peter.pelz\}@ fst.tu-darmstadt.de
}

Keywords: Booster Station, Booster System, Energy Efficiency, Optimization, Technical Operations Research.

\begin{abstract}
Pumps and pumping systems consume about $12 \%$ of the annual electricity production in Europe. A parallel arrangement of two or more pumps is called "booster station". Booster stations meet a varying pressure demand with high energy-efficiency by deactivating individual pumps at smaller loads. Hence, one major difference from single pump units to booster stations is the diversity of control options. Due to the non-linear characteristics of the machines and physical laws we are facing a Mixed-Integer Nonlinear Problem (MINLP). The global optimization of a MINLP requires a specialized solver and a huge amount of time. To reduce the complexity of the problem, we evaluate two possible ways: i) We apply piecewise linearization techniques to the problem in order to gain a Mixed Integer Linear Problem (MILP). The advantage is the vast variety of available solvers and a significant reduction of calculation time. The disadvantage of this method is being less accurate. ii) The enumeration of the integer decisions and the fitting of the characteristic curves by algebraic functions enable us to reduce the complexity of the problem to a simple Nonlinear Problem (NLP). This technique allows us to keep the accuracy of the physical laws, but has the big drawback of the enumeration time. The consecutive use of two global optimization solvers combines the advantages of the formerly mentioned approaches, but we lose the guarantee for global optimality.
\end{abstract}




\section{INTRODUCTION}

Pumps and pumping systems consume about $10 \%$ of the annual electricity production in Europe [1]. The European Union aims for the usage of energy efficient machines to gain energy savings. These energy efficient machines are the first step towards reducing the energy consumption of pumping systems. Various eco-design directives have been and will be carried out in order to ban machines with low efficiency from the market [2].

The second step must be the efficient use of these excellent machines. A parallel arrangement of two or more pumps is called "booster station". Booster stations meet a varying pressure demand with high energy-efficiency by deactivating individual pumps at smaller loads. They cover a wide performance map and are flexible machines with widespread possibilities of usage in industrial and residential applications, e.g. chemical processing plants or water supply in skyscrapers.

The design and control of a booster station offer manifold degrees of freedom. The power input of a booster station in one point of duty depends on more than one design or control variable. Global optimization programs offer help for tuning the mixture of continuous and discrete variables, so that the power input of the booster station becomes minimal.

The aim of our research is to make optimization programs available for engineering use. A modeling framework and exchangeable input data enable us to quickly gain a control strategy and design information from measured characteristics.

\section{TECHNICAL SPECIFICATIONS}

\subsection{Working Principle of a Booster Station}

The technical task of a booster station is to promote water in a piping network. Therefore the pressure is increased to overcome geodesic differences or pressure loss by dissipation. Figure 1 shows the connection scheme of the booster station. Multiple pumps, in most applications of the same type, are connected in parallel. The incoming water flows from the suction pipe, through the single pump units, into the pressure pipe. The pressure at the outlet is always higher than at the inlet. The load of the booster station is given by the total volume flow $Q_{t o t}$ and the pressure head $\Delta H$. To avoid reverse flow, a check valve is installed behind each pump.

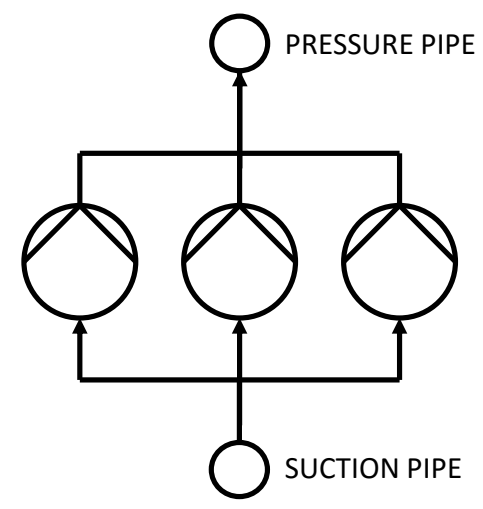

Figure 1: Connection scheme of a booster station. 
The parallel arrangement has two major reasons: Firstly, one is able to avoid heavy part load in the pumps by deactivating single pumps. Secondly, if one pump fails, it is still possible to use the others for promoting water. Other topologies, especially the direct connection of pumps in series, are generally possible and useful [3], but the application of a control strategy in between the considered points of duty is very difficult. Thus, we do not consider this topological option in our paper.

\subsection{Pump Characteristics}

To describe a pump's field of operation, four parameters are relevant: The volume flow $Q$, the pressure head $\Delta H$, the rotational speed $n$ and the power input $P$. The actual point of duty is determined exactly by any two of these parameters for strictly monotonic characteristics. This condition is fulfilled for the relevant operating range for any pump and in this paper for the whole operating range. Figure 2 shows the characteristics of a single pump.

The manufacturer describes the characteristics in his catalogue based on measurement data. For the reference rotational speed $n_{\text {ref }}$ the pressure head and volume flow of the pump are varied in a test rig and the values as well as the corresponding power input are measured. Following industrial standards the unit to measure the pressure head is meter water column $(m W C)$. Table 1 gives the measurement points for the pump type A considered in this paper.
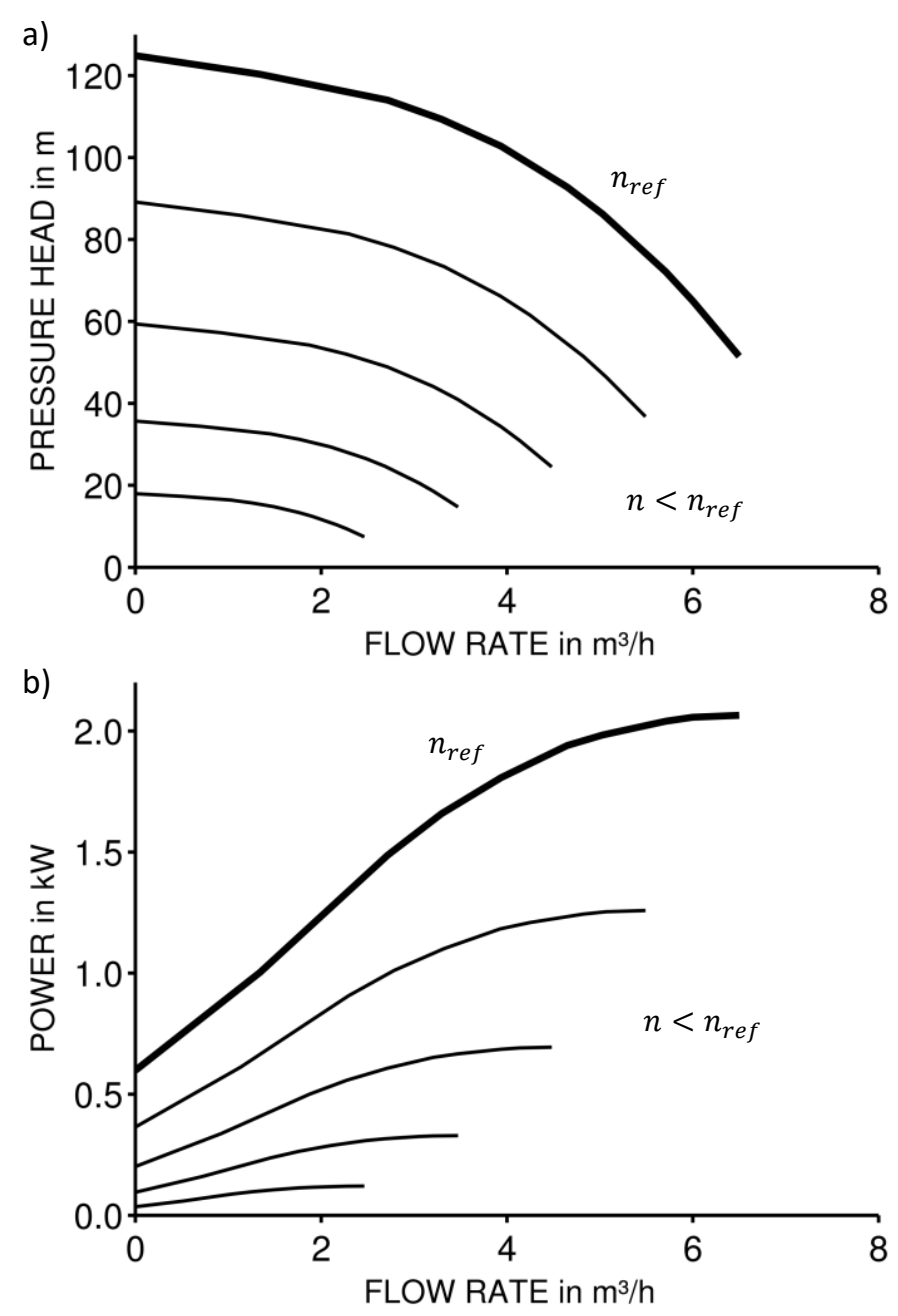

Figure 2: Characteristics of pump type A. 


\begin{tabular}{ccc}
\hline $\begin{array}{c}\text { Flow Rate in } \\
\mathrm{m}^{3} \mathrm{~h}^{-1}\end{array}$ & $\begin{array}{c}\text { Pressure head } \\
\text { in } \mathrm{mWC}\end{array}$ & $\begin{array}{c}\text { Power Input } \\
\text { in } \mathrm{kW}\end{array}$ \\
\hline 0.0000 & 124.87 & 0.5981 \\
1.3421 & 120.31 & 1.0031 \\
2.7176 & 114.00 & 1.4878 \\
3.2971 & 109.33 & 1.6588 \\
3.9364 & 102.76 & 1.8072 \\
4.6499 & 92.78 & 1.9402 \\
5.0308 & 86.19 & 1.9832 \\
5.7083 & 72.11 & 2.0403 \\
6.0010 & 64.99 & 2.0567 \\
6.5000 & 51.49 & 2.0649 \\
\hline
\end{tabular}

Table 1: Measurement points for pump type A.

Many market available pumps are able to alter their rotational speed in the interval $n_{\min } \leq$ $n \leq n_{\text {max }}$. To calculate the characteristics for altering rotational speed, the scaling laws hold:

$$
\begin{gathered}
Q(n)=\left(\frac{n}{n_{\text {ref }}}\right) Q_{\text {ref }}, \\
\Delta H(n)=\left(\frac{n}{n_{\text {ref }}}\right)^{2} \Delta H_{\text {ref }}, \\
P(n)=\left(\frac{n}{n_{\text {ref }}}\right)^{3} P_{\text {ref }} \frac{1-\left(1-\eta_{\text {opt }}\right)\left(\frac{n}{n_{\text {ref }}}\right)^{-0.1}}{\eta_{\text {opt }}} .
\end{gathered}
$$

The scaling law for the power characteristics is expanded by an empirical correction term in order to account for the efficiency decrease due to decreasing rotational speed, wherein $\eta_{\text {opt }}$ stands for the maximum hydraulic efficiency at reference speed. Usually, but not necessarily, the maximal rotational speed of a pump is used as reference value.

\subsection{Operational Mode}

The control variable for the operation of the booster station is the pressure in the pressure pipe. The controller adjusts the rotational speed of the pumps and switches pumps on and off. While the rotational speed is a continuous variable which could be controlled, e.g. by a PID controller, the operational status is a discrete variable that causes a discontinuous transition. To react on this discontinuity the designer implements a control strategy.

This strategy may be devised by many different approaches and with various objectives. A simple approach takes the maximum rotational speed of a pump into account. All active pumps are running at the same rotational speed. Whenever the active pumps cannot fulfil the load at maximum rotational speed the controller turns on an additional pump and adjusts the rotational speed of all pumps. This simple approach guarantees the functionality of the system, but does not consider the power input.

Another option considers the power input. A pump's efficiency is not constant over the volume flow. The degrees of freedom for the control of the booster station are the number of active pumps and the rotational speed of any active pump. The pressure difference over all active pumps must be equal. The total volume flow is distributed between the pumps depend- 
ing on the pump type and the rotational speeds. We will optimize the setting of the control variables, so that the total power input $P_{t o t}$ of the system becomes minimal.

\section{MATHEMATICAL MODEL}

We present different approaches for calculating a design as well as a control strategy for booster stations from the characteristics of the machines. While the rotational speed of a pump is controlled continuously within the technical available range, the topological layout and number of activated pumps are discrete decisions. Due to the non-linear characteristics of the machines and physical laws we are facing a Mixed-Integer Nonlinear Problem (MINLP).

To simplify the MINLP in order to gain a quicker solution we examine two different approaches: We apply piecewise linearization techniques to the problem in order to gain a Mixed-Integer-Linear-Problem (MILP). In the second approach the fixation of the integer decisions and the fitting of the characteristic curves by algebraic functions enable us to reduce the complexity of the problem to a simple Nonlinear Problem (NLP).

\subsection{Pump Characteristics}

Appropriate modelling of pump characteristics is very important for the optimization program. From the characteristics measurement we gain several discrete data points for the operation of the pump at one specific rotational speed. Thus, we have to estimate values in between these points and to expand the data to create the field of operation. In general we have two different possibilities for this estimation: (i) A fitting function or (ii) piecewise linear interpolation. Both options are described in detail in the following:

(i) We use a polynomial function $2^{\text {nd }}$ to $5^{\text {th }}$ degree to fit the reference curve of the pump. The higher the degree of the polynomial function, the better the measured points and the shape of the curve is met. The lower the degree of the polynomial function, the simpler becomes the resulting optimization program. For the pump in our study, we decided for a $3^{\text {rd }}$ degree polynomial to model the pressure characteristics and a $4^{\text {th }}$ degree polynomial to model the power characteristics. The polynomial coefficients are given in Table 2:

$$
\begin{gathered}
\Delta H_{r e f}\left(Q_{r e f}\right)=a_{H} Q_{r e f}{ }^{3}+b_{H} Q_{r e f}{ }^{2}+c_{H} Q_{r e f}+d_{H}, \\
P_{r e f}\left(Q_{r e f}\right)=a_{P} Q_{r e f}^{4}+b_{P} Q_{r e f}^{3}+c_{P} Q_{r e f}{ }^{2}+d_{P} Q_{r e f}+e_{P} .
\end{gathered}
$$

\begin{tabular}{ccc|ccc}
\hline \multicolumn{2}{c|}{ Coefficient } & Value & \multicolumn{2}{c}{ Coefficient } & Value \\
\hline$a_{H}$ & in $\mathrm{mWC} \mathrm{h}^{3} \mathrm{~m}^{-9}$ & -0.2448 & $a_{P}$ & in $\mathrm{kW} \mathrm{h}^{4} \mathrm{~m}^{-12}$ & 0.001357 \\
$b_{H}$ & in $\mathrm{mWC} \mathrm{h}^{2} \mathrm{~m}^{-6}$ & 0.3421 & $b_{P}$ & in $\mathrm{kW} \mathrm{h}^{3} \mathrm{~m}^{-9}$ & -0.02259 \\
$c_{H}$ & in $\mathrm{mWC} \mathrm{h} \mathrm{m}^{-3}$ & -3.197 & $c_{P}$ & in $\mathrm{kW} \mathrm{h}^{2} \mathrm{~m}^{-6}$ & 0.09047 \\
$d_{H}$ & in mWC & 124.9 & $d_{P}$ & in $\mathrm{kW} \mathrm{h}^{1} \mathrm{~m}^{-3}$ & 0.2196 \\
& & & $e_{P}$ & in kW & 0.59811 \\
\hline
\end{tabular}

Table 2: Polynomial coefficients for pump type A.

For altering the rotational speed the scaling laws (equations (2) and (3)) hold. By using additionally equation (1) we derive:

$$
\Delta H(Q, n)=\left(\frac{n_{r e f}}{n}\right) a_{H} Q^{3}+b_{H} Q^{2}+\left(\frac{n}{n_{r e f}}\right) c_{H} Q+\left(\frac{n}{n_{r e f}}\right)^{2} d_{H}
$$




$$
\begin{gathered}
P(Q, n)= \\
{\left[\left(\frac{n_{r e f}}{n}\right) a_{P} Q^{4}+b_{P} Q^{3}+\left(\frac{n}{n_{r e f}}\right) c_{P} Q^{2}+\left(\frac{n}{n_{\text {ref }}}\right)^{2} d_{P} Q+\left(\frac{n}{n_{\text {ref }}}\right)^{3} e_{P}\right]} \\
\frac{1-\left(1-\eta_{\text {opt }}\right)\left(\frac{n}{n_{\text {ref }}}\right)^{-0.1}}{\eta_{\text {opt }}} .
\end{gathered}
$$

Due to the shape of the characteristic curves of a pump (cf. Figure 2) and the nonlinear scaling laws this technique necessarily leads to a nonlinear model (MINLP or NLP).

(ii) The reference measurements for the pump are given as triples $\left(Q_{r e f}, \Delta H_{r e f}, P_{r e f}\right)$ for the reference rotational speed. For altering the rotational speed the scaling laws hold once again. But in this case we discretize the rotational speed on the permitted interval and explicitly scale the triples of measurement points for these discrete values. As stated in Chapter 2.2, only two of the four values for volume flow, pressure head, power input and rotational speed are independent from each other. If the point of duty is fixed in the $Q-\Delta H$-plane, the values for rotational speed and power input are directly dependent.

To interpolate between the points we use the aggregated convex combination (ACC) described by Vielma et al. [4]. The closer the intervals between the discrete values for the rotational speed are, the smaller is the possible linearization error, but the larger becomes the optimization problem.

The choice for a pump model directly influences the possible options for the final optimization program: Linear interpolation in an optimization program requires the use of binary or integer variables. Hence the resulting problem will include discrete variables (MINLP or MILP).

\subsection{Flow Model}

We model fluid systems as a mathematical flow graph $G(V, E)$. The edges $E$ of the graph represent technical components, in this particular case the pumps $K$, or simple connections. The vertices $V$ are used to connect the edges [5]. Each edge transfers a volume flow $Q$. In each vertex the pressure $H$ is calculated. For every pump edge a power input $P$ and the rotational speed $n$ is calculated additionally.

Depending on the optimization scope and the program type, two different formulations for the load case are applicable: (i) each pump type is represented as one edge of the graph or (ii) each pump is represented by an edge of the graph.

(i) If one edge of the flow graph represents a number of pumps of the same type, one further premise applies: The rotational speed for all pumps of this type is the same. We introduce an integer variable $x$ for each pump type that represents the number of active pumps for this pump type and modify the requirement for the load case:

$$
Q_{\text {tot }}=\sum_{\{i \text { in } K\}} x_{i} Q_{i} .
$$

Due to the parallel arrangement the volume flow for all pumps of one type equals the volume flow for a single pump multiplied by $x$. The required pressure head of the booster following from the given input and output pressure is not influenced by the variable $x$ and still accounts for all pumps. Parallel pumps must have the same pressure head. 


$$
\forall i \in K: H_{i, \text { input }}+\Delta H_{i}=H_{i, \text { output }}
$$

If the range of $x$ includes 0 , the pump type can be deactivated. The pressure head and the rotational speed, following from the pump characteristics model, will still be calculated, but the energy consumption of the pump vanishes.

The correlation of the power input for multiple pumps is the same as of the volume flow and also uses variable $x$.

$$
P_{\text {tot }}=\sum_{\{i \text { in } K\}} x_{i} P_{i}
$$

For purely nonlinear problem formulations without integer variables (NLP) the variable $x$ may be transformed into a parameter. Enumeration is a possible approach to find the optimal number of active pumps. In this case only the rotational speeds for the different pump types are the remaining degrees of freedom. For booster stations with only one pump type the optimization problem becomes a feasibility problem.

(ii) The booster station has to promote the total volume flow $Q_{t o t}$, which is given as input parameter, as the sum of the single volume flows in each pump:

$$
Q_{t o t}=\sum_{\{i \text { in } K\}} Q_{i}
$$

Further input parameters for the point of duty are the input pressure and the required pressure head for the booster station. The pressure head (equation (9)) for the booster station accounts for any active pump. To deactivate a specific pump, we introduce a binary activity variable $y$ and use a Big-M-Formulation to modify equation (9) and decouple the pressure at the inlet and outlet of the pump.

$$
\begin{array}{ll}
H_{i, \text { input }}+\Delta H_{i} \leq H_{i, \text { output }}+M(1-y) & \forall i \in K \\
H_{i, \text { input }}+\Delta H_{i} \geq H_{i, \text { output }}-M(1-y) & \forall i \in K
\end{array}
$$

A deactivated pump $\left(y_{i}=0\right)$ has no volume flow or power input. This is realized by forcing all weights $\lambda$ of the linearization (compare [4]) to be zero. Thus the total energy input reads

$$
P_{t o t}=\sum_{\{i \text { in } K\}} P_{i} .
$$

Due to the use of the binary variable, the application of a Big-M-Formulation is only possible in an approach which allows discontinuous variables (MINLP or MILP).

Please note: In the MINLP case, the deactivation model for single pumps is also possible in a manner similar to the approach described in (i): Instead of the integer variable $x$ the binary activity variable $y_{M}$ is used to modify the flow and the power equation of each pump. Pressure rise and rotational speed will be calculated also for the deactivation in this case, but do not influence the optimization program and result. The rotational speed and the power input for all pumps follow from model for the pump characteristics.

If binary variables must be avoided to use the NLP approach, the remaining degrees of freedom for the optimization are the continuous variables for the rotational speed. To find the optimal number of active pumps for this case, an enumeration is a possible approach. 


\subsection{Objective}

The objective for the optimization program is to minimize the total power input of all pumps for the given point of duty.

$$
\min P_{\text {tot }}
$$

\subsection{Optimization Framework}

In order to quickly gain solutions for optimization problems of booster related tasks, we created an optimization framework within the AIMMS software system. The parameters for the optimization, such as different pump types as well as their characteristics or maximum number of pumps, are given as user input, but remain the same for any optimization program. From the user input the flow graph is generated. Furthermore, the framework includes the constraints and variables for all different modelling options. The user chooses which type of optimization program he would like to use and the computer compiles only the relevant part of the model for the user's choice. Afterwards the resulting optimization program is passed to a matching solver. This framework enables us to compare the results for the different modelling approaches and compare them to each other.

The described optimization program finds an optimal control solution for one point of duty. In order to find control guidelines for the whole field of operation, we have to run the optimization model many times with varying load cases. We discretize the pressure and the volume flow and run the program for every point of duty.

\section{RESULTS}

\subsection{Control Strategy for Booster Stations with one Pump Type}

We compare three different approaches to optimize the control strategy of a booster station consisting of three pumps of type A. The first approach is a MILP: We model the pump characteristics by an ACC linearization with 20 intervals for the rotational speed and create one edge for each pump. The second model is an NLP approach, which uses a fitted pump model and one edge for each pump type. The discrete decision for the optimal number of pumps follows from an enumeration and comparison of the results. The third model is a MINLP with the same model as the NLP. Instead of the enumeration the Solver additionally makes a discrete decision for the number of active pumps.

The result for the discrete decision for the number of pumps is the same with all three approaches. Figure 3 shows the optimal control strategy for the booster station. We can identify two reasons for the switch of a pump: (1) For high constant pressure and increasing volume flow the controller increases the rotational speed of the active pumps. Once the maximum is reached, an additional pump is switched on. The decreasing gray lines show the maximum volume flow for fixed pressure head for one, two or three pumps. (2) For low constant pressure and increasing volume flow we find an efficiency argument: An additional pump is switched on, because it reduces the total power input, even though more pumps are working. 


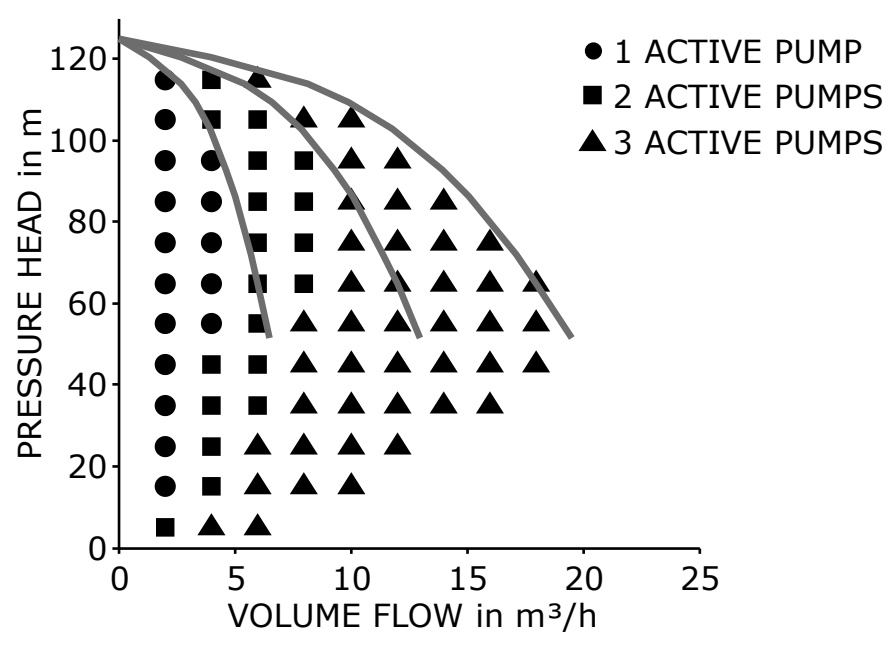

Figure 3: Optimization result for a booster station of three pumps of type A.

All calculations were made on an AMD Phenom ${ }^{\mathrm{TM}}$ II X6 1100T Processor with $3.3 \mathrm{GHz}$ and 16 GB RAM. The applied solvers were CPLEX 12.6.2 for the linear model and BARON 15 for the nonlinear model. The sum of the run times for the different approaches is:

- MILP:

- MINLP:

- NLP + Enumeration: $51.6 \mathrm{~s}$

The MILP is clearly the fastest, while, as expected, the NLP takes the most time. Even though the discrete decision of all the approaches remains the same, the solutions are slightly different: In the MILP results the rotational speed of the active pumps is not always exactly the same. For $\Delta H=75 \mathrm{mWC}$ and $Q=12 \mathrm{~m}^{3} / \mathrm{h}$ the rotational speeds for the three active pumps are $n_{1}=2607 \mathrm{~min}^{-1}, n_{2}=2521 \mathrm{~min}^{-1}, n_{3}=2607 \mathrm{~min}^{-1}$. Even though it's proven, that all these pumps should run at the same rotational speed [6], the optimality gap is small enough to stop the solver. Highest settings for the numerical precision of the solver can't change this. Additionally, the linearization error can only be calculated and reduced [7] but not avoided for the optimization. A nonlinear correction would be necessary to use the results from this approach. The necessary correction is a feasibility problem (see 3.2).

\subsection{Control Strategy for Booster Stations with two Pump Types}

The next considered optimization problem is a booster station with three pumps of type A and one pump of different type. Table 3 shows the measurement points for pump type B. Once again we use the MILP and the MINLP approach as described in 4.1. The NLP approach is not tested anymore, due to the bad performance for the first test case and the increased number of decisions. 


\begin{tabular}{ccc}
\hline $\begin{array}{c}\text { Flow Rate in } \\
\mathrm{m}^{3} \mathrm{~h}^{-1}\end{array}$ & $\begin{array}{c}\text { Pressure head } \\
\text { in } \mathrm{mWC}\end{array}$ & $\begin{array}{c}\text { Power Input } \\
\text { in } \mathrm{kW}\end{array}$ \\
\hline 0.0000 & 123.86 & 0.4597 \\
0.6000 & 120.80 & 0.6289 \\
1.2215 & 114.09 & 0.7930 \\
1.6223 & 107.30 & 0.9069 \\
1.8152 & 103.55 & 0.9673 \\
1.9219 & 101.30 & 0.9887 \\
2.0529 & 86.11 & 1.0242 \\
2.4428 & 72.11 & 1.1013 \\
2.6902 & 75.95 & 1.1334 \\
3.0266 & 59.32 & 1.1545 \\
\hline
\end{tabular}

Table 3: Measurement points for pump type B.

The computer and the solvers stay the same. The summed solver times for the solution of the new problem are:

- MILP: $20.0 \mathrm{~s}$

- MINLP: $654.0 \mathrm{~s}$

One can see that the MILP is much faster than the MINLP. Figure 4 shows the results for the number of active pumps as given by both solvers. The solutions of the solvers differ in six of the 90 feasible cases. The objectives in these points are nearly equal. The reasons for theses slight differences are related to our given reasons for the different rotational speeds in Chapter 4.1. Due to the different model types we gain different solutions.
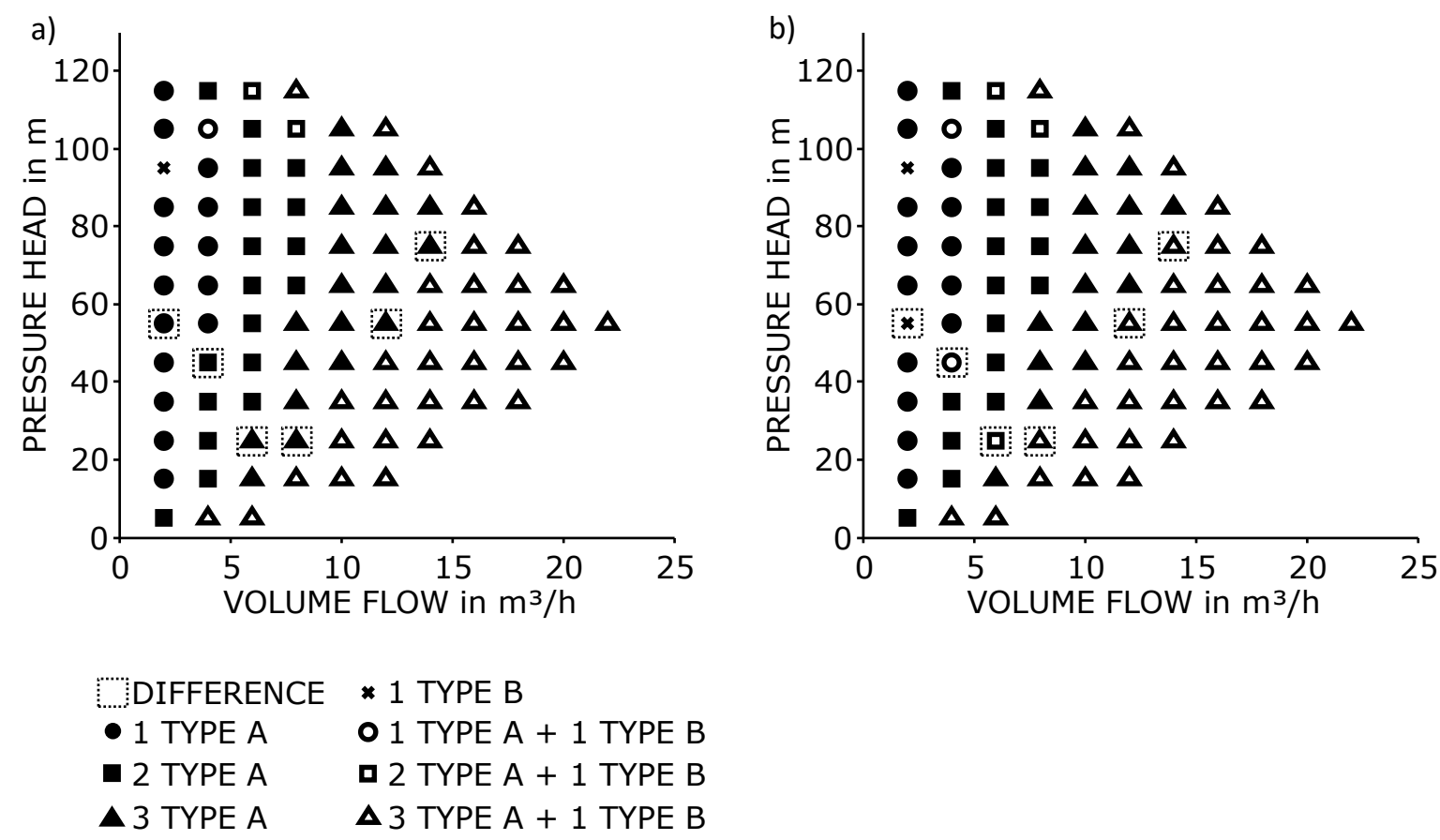

Figure 4: Optimization result for a booster station of three pumps of type A and one pump of type B.

a) MILP, b) MINLP 
Also in this case, we notice different rotational speeds for the active pumps of type A. The inevitable correction of the rotational speeds is not necessarily a feasibility problem anymore. Pumps of different types may but do not have to run at the same rotational speed to minimize the energy input. Thus this might become an optimization problem if pumps of both types are active.

As a next step we combine both methods: We use the MILP to make discrete decision for the active pumps. Afterwards, we use the discrete result as input for the NLP-Solver to gain an optimal and non-linear feasible solution for the original problem. The summed computation time for this approach is $223.6 \mathrm{~s}$, so this approach is much quicker than the MINLP due to the excellent performance of the MILP solver.

\section{CONCLUSIONS}

- Pumping systems and booster stations use a significant amount of the produced electrical energy and thus need to be considered for optimization.

- We presented the technical requirements for the optimization of booster stations.

- Different approaches are suitable for the optimization of the control and layout of a booster station.

- We implemented a framework, which allows us to compare the different approaches and calculate appropriate solutions quickly.

- The effort of building a MILP is the highest, but the solvers work very quickly and deliver good results.

- Finding a nonlinear feasible solution corresponding to a MILP solution is a good alternative for the use of a MINLP solver.

\section{REFERENCES}

[1] Pelz, P. F.: 250 Jahre Energienutzung: Algorithmen übernehmen Synthese, Planung und Betrieb von Energiesystemen, Fachvortrag anlässlich der Ehrenpromotion von HansUlrich Banzhaf, 23.01.2014, Universität Siegen, 2014.

[2] Bidstrup, N.; Teepe, M.; Berge, G.; Ludwig, G.: Extended Product Approach for Pumps, Draft Version, Europump, 2014.

[3] Pelz, P.; Lorenz, U.: As good as can be! TOR plans the energetically optimal fluid system. ErP Special Issue, Delta p, 2013.

[4] Vielma, J.P.; Nemhauser, G.L.: Modeling disjunctive constraints with a logarithmic number of binary variables and constraints; Math. Program. Ser., 2011.

[5] Pöttgen, P.; Ederer, T.; Altherr, L. C.; Pelz, P. F.: Developing a Control Strategy for Booster Stations under Uncertain Load. Applied Mechanics and Materials, 807 pp. 241246. ISSN 1660-9336, 2015.

[6] Groß, T; Pöttgen, P.; Pelz, P. F.: An Analytical Approach for the Optimal Operation of Pumps in Booster Systems, not yet published. 
[7] Geißler, B: Towards Globally Optimal Solutions for MINLPs by Discretization Techniques with Applications in Gas Network Optimization, Dissertation, FriedrichAlexander-Universität Erlangen-Nürnberg, 2011. 